\title{
Intelligent text processing to help readers with autism
}

\author{
Constantin Orăsan, Richard Evans and Ruslan Mitkov
}

\begin{abstract}
Autistic Spectrum Disorder (ASD) is a neurodevelopmental disorder which has a life-long impact on the lives of people diagnosed with the condition. In many cases, people with ASD are unable to derive the gist or meaning of written documents due to their inability to process complex sentences, understand non-literal text, and understand uncommon and technical terms. This paper presents FIRST, an innovative project which developed language technology (LT) to make documents more accessible to people with ASD. The project has produced a powerful editor which enables carers of people with ASD to prepare texts suitable for this population. Assessment of the texts generated using the editor showed that they are not less readable than those generated more slowly as a result of onerous unaided conversion and were significantly more readable than the originals. Evaluation of the tool shows that it can have a positive impact on the lives of people with ASD.
\end{abstract}

Mathematics Subject Classification (2010). Primary 97R40; Secondary $91 \mathrm{~F} 20$.

Keywords. Language technology, Autism Spectrum Disorder, Text simplification, Text accessibility.

\section{Introduction}

Autistic Spectrum Disorder (ASD) is a neurodevelopmental disorder characterised by qualitative impairment in communication and stereotyped repetitive behaviour. It is a serious disability that affects approximately 60 people out of every 10,000 in the EU. People with ASD usually have language deficits with a life-long impact on their psychosocial functioning. These deficits are in the comprehension of speech and writing, including misinterpretation of literal meanings and difficulty understanding complex instructions [39]. Complex sentences, referential expressions, uncommon or technical words and figures of speech also constitute obstacles to proper understanding of texts for people with ASD. In many cases, people with ASD are unable to derive the 
gist or meaning of written documents [41, 42, 23]. The difficulties in reading comprehension that ASD causes represent a significant barrier to inclusion. People require access to written material for many purposes and in many contexts, from searching for employment opportunities and obtaining information to support their education to communicating by email or learning about local entertainment or news. Several studies have indicated a link between reading comprehension (and more generally, literacy) and access to education, employment, culture, and communication [9, 43].

This paper presents FIRST, an innovative project which developed language technology (LT) to make documents more accessible to people with ASD. OpenBook, the conversion software developed in this project, ${ }^{1}$ is able to automatically detect a range of language phenomena which are problematic for people with ASD and replace some of them to make the text more comprehensible. It also aims to simplify complex structure in the text and clarify ambiguity. Not relying purely on textual changes, the conversion software adds illustrative pictures to documents and concise document summaries. Evaluation of LT carried out in the project revealed that the language processing components developed make a relatively large number of errors when dealing with unrestricted text. This is a problem given that the end users of the tool have low tolerance for ungrammatical and erroneous text which may be generated by LT components. For this reason, the OpenBook tool offers powerful post-editing options to carers to enable them to prepare texts for end users. In this way, all the changes made to a document can be supervised by carers who will ensure that the simplification is correct and the appropriate type of simplification is applied for a particular user. All this is in addition to the personalisation features embedded in the software.

Given the size of the project and the variety of topics covered during the three years it ran for, we cannot provide a detailed account of all the research we carried out. Instead, in this paper we provide an overview of the main achievements of the project with references to papers that provide more details. This paper is structured as follows: Section 2 reviews several similar projects and a survey of the most relevant literature. Section 3 presents a brief overview of FIRST and the language technology integrated in OpenBook, followed by an evaluation of the tool in Section 4. The paper ends with discussion and conclusions.

\section{Related work}

The challenge of text simplification has been addressed in several lines of research since the 1990s. Text simplification systems have been developed to rewrite text using various lexical $[61,16,30,6,57,8]$, syntactic $[11,10$, $51,13]$, and other [53, 58] transformation operations and components for the generation of assistive content such as definitions [19], images [7, 3], and summaries [3]. In previous work, text simplification has been used to improve

\footnotetext{
${ }^{1}$ http://openbook.net
} 
the accuracy of NLP applications in areas such as dependency parsing [26] biomedical information extraction [49, 21], semantic role labeling [55], and machine translation $[12,40]$.

Of more relevance to this paper, text simplification methods have also been developed with the goal of facilitating text processing by various populations of readers, including people with poor literacy [52] or numeracy [5], people with aphasia [35], dyslexia [48], autism [22], people who are non-native speakers [1, 45], and children and language learners [29].

Max [35] described the use of a syntactic parser for sentence rewriting to facilitate the reading comprehension of people with aphasia. In the PSET project, Canning [10] implemented a system which exploits a parser in order to rewrite compound sentences as sequences of simple sentences and to convert passive sentences into active ones. One weakness of this approach is that it depends on high levels of accuracy and granularity of automatic syntactic analysis. Research has shown that the accuracy of parsers is inversely proportional to the length and complexity of the sentences being analysed [36]. These are often the sentences for which simplification is most required.

More recently, the availability of resources such as Simple Wikipedia (SW) has enabled text simplification to be included in the paradigm of statistical machine translation $[14,56]$. In this context, translation models are learned by aligning sentences in Wikipedia with their corresponding versions in SW [63]. Manifesting Basic English [44], the extent to which SW is accessible to people with reading difficulties has not yet been fully assessed. Effective SMT relies on the availability of representative pairs of texts in their original and converted forms. At present, these resources are scarce and are often designed with a particular readership in mind, such as children [4, 59], people with Down's syndrome [8], or people at particular reading grade levels [46]. As a result, there are currently only a limited number of contexts in which SMT approaches are likely to be effective. Xu et al. [59] are critical of the use of Simple English Wikipedia to support SMT-based text simplification.

The field of automatic text summarisation also includes approaches that exploit text simplification processes. For example, Cohn and Lapata [13] present a syntactic tree-to-tree transduction method to filter non-essential information from syntactically parsed sentences. This compression process often reduces the syntactic complexity of those sentences. One advantage of this approach is that it can identify elements for deletion in the absence of explicit lexical/punctuational markers. However, these methods are "destructive" in the sense that information is deleted rather than preserved as a result of compression. Although some information loss is inevitable in text simplification, the method that we exploit in the FIRST project is designed not to automatically delete parts of input sentences.

This survey of related work has demonstrated that the field of text simplification has received a significant amount of interest from researchers in computational linguistics. However, there are very few projects which brought 
together such researchers with end users who could benefit directly from the research at a scale that the FIRST project did.

\section{Overview of the project and the language technology}

The FIRST project was funded by the EC through its FP7 ICT work programme and addressed the objective concerning smart and personalised inclusion. The purpose of the project was to implement an advanced ICT-enabled solution for the empowerment of people with disabilities who are at risk of social exclusion as a result of their low literacy, resulting from cognitive and mental impairments. In line with this, the main aim of the project was to implement, deploy, and evaluate intelligent technology to support the authoring of accessible content in Bulgarian, English, and Spanish for users with ASD with a view to widening inclusion and empowerment in Europe.

The project was coordinated by the University of Wolverhampton and consisted of nine partners representing all the relevant stakeholders: language technology experts (University of Wolverhampton, UK; University of Alicante, Spain and University of Jaen, Spain), clinical partners who work directly with people with ASD (Central and North West London NHS Foundation Trust, UK; Deletrea, Spain and Parallel World, Bulgaria), software developers (iWeb technologies, UK and Kodar, Bulgaria) and an organisation which promotes the rights of people with ASD (Autism Europe, Belgium).

One of the challenges that became quite obvious from the beginning of the project was the fact that there is no clear description of the needs that people with ASD have. Therefore one of the first tasks undertaken in the project was to derive a detailed and accurate description of the requirements of users with reading difficulties. A summary of the findings are presented in Section 3.1 and they informed the choice of the LT integrated in the tool. On the basis of the analysis carried out, we split the LT processing into three main components: a structural complexity processor (Sections 3.3), a meaning disambiguator (Section 3.4) and a personalised document generator (Section 3.5). These components are integrated with the user interface and backend services using a three-tier architecture (Section 3.2).

\subsection{User requirements}

One of the first tasks undertaken in the project was to understand the needs of people with ASD. In light of this, our first objective was to derive a detailed and accurate description of the requirements of users and to gain an understanding of the most significant obstacles to reading comprehension. We also tried to learn how best to convert texts containing such obstacles into a form suitable for end users. The resulting specification needed to be granular enough to support practical design of the core LT in the project.

The user requirements were derived on the basis of an extensive literature survey and as a result of consultations with end users and intermediaries. For the latter, a sample of 37 children (aged 12-16) and 57 adults 
(aged 16+), meeting strict DSM-IV-TR criteria for $\mathrm{ASD},{ }^{2}$ with $\mathrm{IQ}>70$, whose mother tongue was Bulgarian, English or Spanish, with the ability to read and with basic computer skills, were consulted. A pilot study with 25 Spanish participants was carried out first in order to improve the design of questionnaires and face-to-face interviews. The questionnaires were used to analyse specific reading comprehension problems by presentation of a series of texts followed by closed questions related to the content of those texts. Interviews and open questions were used to explore subjective and qualitative information (perception of difficulties regarding reading comprehension and preferences). Responses to the questions consisted of judgements on a 5 point rating scale indicating difficulty of comprehension. Responses to open-ended questions raised several topics that had not been considered in the design of the study.

Intermediaries were also presented with a questionnaire in order to complement the information collected from end-users. The questionnaires focused mainly on their perception of the reading difficulties that they have and the strategies used to overcome them. All the interviews and questionnaires were handled by the clinical partners in the project who had expertise in recruitment of participants with ASD and organisation of meetings with our focus group.

The results of the analysis were categorised as (a) linguistic obstacles to be removed, (b) preferences regarding the format of the output document, (c) preferences regarding the look and feel of the interface and (d) users suggestions of additional features that may be incorporated into the tool. Overall, the results obtained were in line with the findings of similar previous studies. The study conducted in FIRST is innovative with regard to the wide range of areas about which end users were consulted, the types of participant, and the qualitative information obtained from those participants. A set of user requirements was derived from the data obtained and these requirements were then categorised according to the type of LT service addressing each of them (structural complexity processor, meaning disambiguator, personalised document generator). Each user requirement was associated with an editing operation ("assistive element") expected to remove the obstacle to reading comprehension to which the user requirement pertained ("obstacle"). The main user requirements for each type of processing attempted in the project are summarised in subsections 3.3-3.5. More details of those requirements are presented by Martos et al. [34] and González-Navarro et al. [24].

In addition to providing the specifications of the LT components, issues that are specific to our end users such as data protection requirements, including privacy, safety, security, and identity management also had to be addressed. Ethical approval was obtained for all interactions with end users, regardless of whether their involvement was for the purpose of establishing the user requirements, or evaluating the system.

\footnotetext{
${ }^{2}$ http://behavenet.com/apa-diagnostic-classification-dsm-iv-tr
} 


\subsection{Architecture of the system}

OpenBook, the system developed in the FIRST project, features a three-tier architecture which enabled us to develop a modular system that can be easily maintained, scaled up and extended. By using loose coupling we are able to easily replace modules with better ones over time. The three tiers are:

- Presentation tier: controls interaction between users and the system via a graphical user interface. While designing the user interface, best practice in terms of accessibility, the Windows User Experience Interaction Guidelines $^{3}$ and Designing UX for Apps were taken into consideration ${ }^{4}$.

- Integration engine: acts as a business tier and provides interaction between the presentation tier and the LT modules. It also controls user management, access control level, error and logging management.

- Resources tier: contains the language technology services which are responsible of identifying and processing obstacles to reading comprehension. The outputs of these LT services are consumed by an Aggregator Web Service which combines different types of annotation and deals with possible conflicts between them. The aggregator also communicates with the integration engine. The GATE document format [15] was used for communication between different web services. The GATE document format was selected because it allows re-use of existing tools, makes the system easily extensible, and provides flexibility.

Users of the tool are able to customise it depending on their needs. They can affect both the look and feel of the interface, and the way the LT components run. The next sections present in more detail the types of language processing attempted in the project.

Given the difficulties encountered in automatic processing of language, OpenBook features two interfaces. The first is a powerful editor that gives intermediaries the possibility to simplify texts using a host of LT components. Figure 1 shows this interface during the process of inserting an image to represent the term windfarms. The assumption is that intermediaries can easily identify mistakes made by the computer and correct them. Figure 2 presents the interface for end users which provides access to a more limited number of LT components and is meant mainly for reading documents prepared by intermediaries.

\subsection{Processing structural complexity}

In FIRST, we focus on structural complexity at the morphological and syntactic levels of language. The complexity of discourse structure is addressed to some extent by the meaning disambiguator (Section 3.4), which facilitates human processing of a limited set of discourse relations such as coherence and anaphora when reading. Our user requirements analysis led to the derivation of a set of user requirements of various levels of importance. Table 1 lists those

\footnotetext{
${ }^{3} \mathrm{http}: / /$ msdn.microsoft.com/en-us/library/windows/desktop/aa511258.aspx

${ }^{4}$ http://msdn.microsoft.com/en-us/library/windows/apps/hh779072.aspx
} 


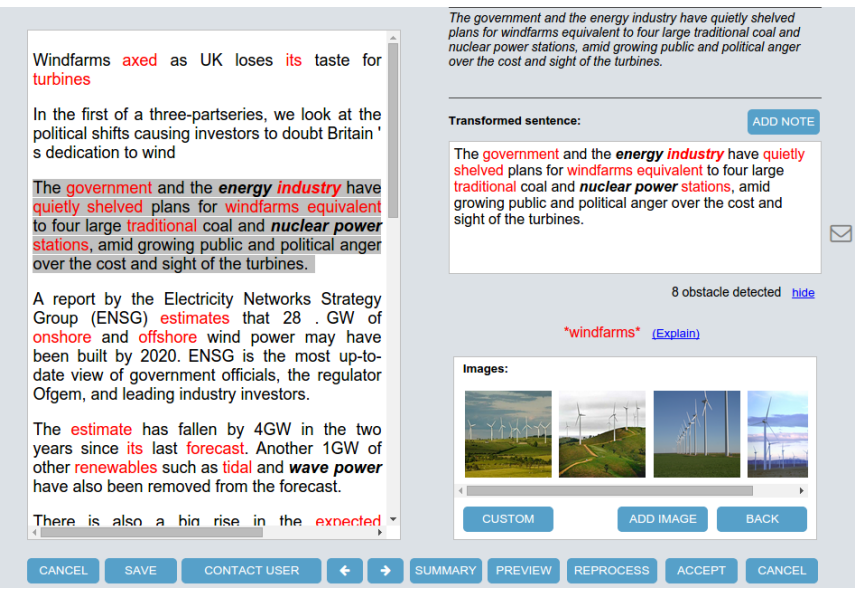

Figure 1. The interface for intermediaries

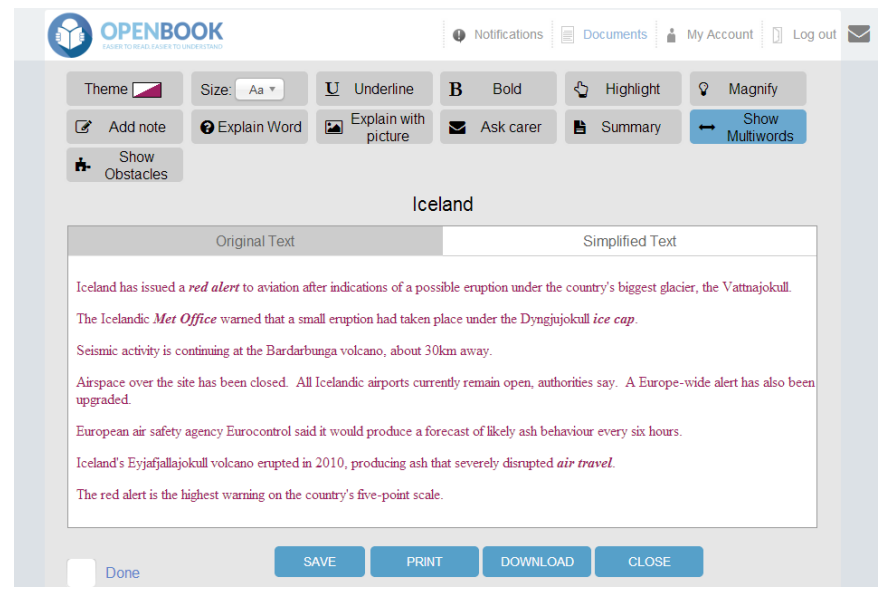

FiguRE 2. The interface for end users

with the highest level of importance. A few requirements linked to presentation (e.g. avoid cutting paragraphs at the end of page) and some that were dealt with by the meaning disambiguator (e.g. substitute rare conjunctions by more common ones) are omitted for brevity.

Examination of the user requirements revealed that many of them cannot be addressed automatically with sufficient accuracy using existing language technology. This is because either the existing research is not advanced enough to address the problems identified or, for languages such as Bulgarian and Spanish, there are no resources which can be used to adapt existing LT to these languages. 


\begin{tabular}{|l|l|l|}
\hline Obstacle & Text simplification operation & ID \\
\hline $\begin{array}{l}\text { Multiple copulative } \\
\text { coordinated clauses }\end{array}$ & Substitute with sentences divided by periods. & UR301 \\
\hline $\begin{array}{l}\text { Subordinate adjectival/ } \\
\text { relative clause }\end{array}$ & $\begin{array}{l}\text { Substitute by adjective or extract and rephrase as } \\
\text { a sequence of sentences. }\end{array}$ & UR302 \\
\hline Explicative clauses & Remove explicative clause & UR303 \\
\hline $\begin{array}{l}\text { Adverbial clause after } \\
\text { main clause }\end{array}$ & Place adverbial clauses before main clause. & UR307 \\
\hline $\begin{array}{l}\text { Conditional clause after } \\
\text { main clause. }\end{array}$ & Place conditional clauses before main clause. & UR308 \\
\hline Long sentences & Rephrase into shorter sentences (less than 15 words) & UR309 \\
\hline $\begin{array}{l}\text { Semicolon and } \\
\text { suspension points }\end{array}$ & Avoid the use of semicolon and suspension points. & UR310 \\
\hline $\begin{array}{l}\text { Sentences in passive } \\
\text { voice }\end{array}$ & Use active voice. & UR313 \\
\hline $\begin{array}{l}\text { Sentences with double } \\
\text { negatives }\end{array}$ & $\begin{array}{l}\text { Avoid sentences containing negatives and double } \\
\text { negatives }\end{array}$ & UR314 \\
\hline
\end{tabular}

TABLE 1. User requirements relating to the processing of structural complexity

Most of the work carried out in this project on processing structural complexity focused on reducing the syntactic complexity of English sentences. In text, conjunctions, complementisers, wh-words, punctuation marks, and pairs consisting of a punctuation mark followed by one of these types of word are signs indicating the presence of syntactic complexity in a sentence. These signs link clauses and phrases together in coordination and also bound subordinate phrases and clauses embedded in complex constituents. To automatically reduce the syntactic complexity of English texts, we developed a method which can automatically identify and classify signs of syntactic complexity using a machine learning approach and rewrite complex sentences using a predefined set of rules. The method was designed in such a way that it can be adapted to other languages. Unfortunately, this was not possible during the FIRST project due to time constraints. Bulgarian and Spanish complex sentences were rewritten using a limited number of rules applied to the output of syntactic parsing. For this reason, coverage is limited.

As noted by Siddharthan [51], text simplification can be viewed as comprising three processes: analysis, transformation, and post-editing. In previous work, Evans [21] described a rule-based method for sentence rewriting. The main contributions of this method were a new approach to automatic sentence analysis and a method for rewriting sentences on the basis of that analysis. This analysis includes tokenization of input texts to enable identification of sentences, words, and potential coordinators, PoS tagging, and a machine learning method to categorize potential coordinators according to their specific coordinating functions. The method proved useful as a preprocessing step in biomedical information extraction [21]. We took the same approach in the FIRST project, but focused on a wider set of signs of syntactic complexity and addressed a wide range of types of both coordination and subordination. The method was also developed for a wider variety of texts. 


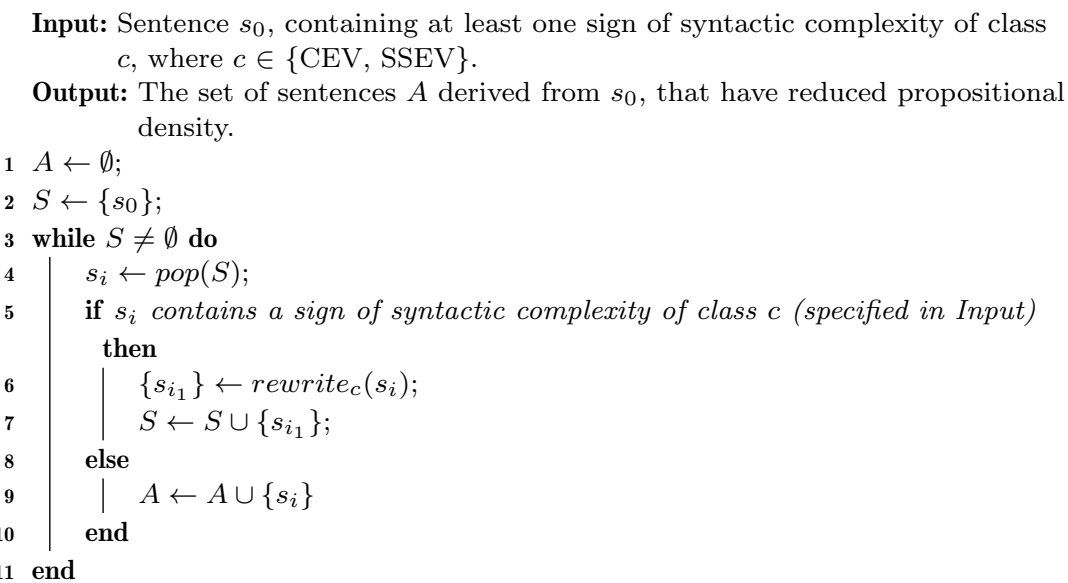

Algorithm 1: Syntactic simplification algorithm

The rest of this section briefly presents the processing applied to English sentences. These automatic processes address user requirements UR301-303 and UR309-310 (Table 1). Our method exploits Algorithm 1.

3.3.1. Identification of signs of syntactic complexity. The identification of signs of syntactic complexity is achieved by using a supervised tagging approach which builds statistical models for predicting the functional class of signs of syntactic complexity. CRF tagging models [31, 54] were built and their intrinsic performance analysed. This approach was employed because, during development, we found that sequence based CRF tagging models provide better performance in the automatic tagging of signs than methods in which each sign is tagged independently of other signs in the same sentence. The mean accuracy of the best model, applied to texts of three different registers (health, literature, and news), was $82.06 \%$. More details on our approach and its evaluation are presented in the paper by Dornescu et al. [18]. ${ }^{5}$

Information about the functions of different signs of syntactic complexity, in combination with patterns used to detect passive sentences, and gazetteers of complex and rare conjunctions were also used to provide carers with reports on the types of syntactic complexity detected within a sentence. These reports can be used to inform carers about how to rewrite sentences in order to make them more accessible. An example can be seen in Table 2 .

3.3.2. Sentence rewriting. A rule-based approach was developed to convert compound sentences into sequences of sentences containing no coordinate clauses, and sentences containing complex noun phrases into sentences containing simple noun phrases. In our approach, sentences were tagged with information on the parts of speech of words and the syntactic functions of the signs of syntactic complexity that they contain. Sentence rewriting rules

\footnotetext{
${ }^{5} \mathrm{~A}$ demo of the English sign tagger is available at http://rgcl.wlv.ac.uk/demos/SignTagger WebDemo/
} 
When processing the sentence

The judge, Richard Walker, has ruled as a matter of law that the words are libellous, and the jury is being asked to decide the scale of damages, which Mr Burstein's solicitor advocate said should be between 20,000 and 50,000.

the system generates a report such as

This sentence contains:

- 2 embedded clauses. These sentences may contain multiple facts. Texts are easier to read when each sentence contains one fact:

- ... as a matter of law [that] the words are...

- ... the scale of damages[, which] Mr Burstein's solicitor...

- 1 other embedded phrase. These sentences may contain multiple facts. Texts are easier to read when each sentence contains one fact.

- ... The judge[,] Richard Walker, has...

- 1 pair of linked clauses. These sentences may contain multiple facts. Texts are easier to read when each sentence contains one fact.

- ...the words are libellous[, and] [the jury is being asked...]

- 1 passive verb in a subordinate clause. These sentences contain multiple facts. Texts are easier to read when each sentence contains a single fact. When converting this sentence to a more readable form, try to ensure that the correct agent of the embedded verb is explicitly mentioned.

- ... libellous, and the jury [is being asked] to decide the scale of...

TABLE 2. Example of sentence analysis produced by the system

were then applied iteratively, each rule triggered by matching patterns in the tagged sentences. By counting how many times the application of a rule led to the generation of correct output we were able to determine the accuracy of the simplification process. Overall, the rules used to rewrite sentences containing compound clauses have an accuracy of 0.699 . The rules used to rewrite sentences containing bound relative clauses have an accuracy of 0.583 . The two primary sources of error were the specificity of the rules, which limits their coverage and the inability of the method to discriminate between conjunctions and commas linking coordinate bound relative clauses and those linking independent clauses [22].

Evaluation of the English structural complexity processor showed that the LT developed in the FIRST project performs at a level that compares favourably with the state of the art. However, in contexts where end users have a low tolerance for errors, direct access to some of these components should be limited. In FIRST, intermediaries have direct access to the full set of LT components via the carers' interface. They are the ones who process texts automatically and then post-edit the output to generate a more accessible form of the input text that can be consumed directly by end users, in this way addressing the errors introduced by LT.

The overall impact of the processing of syntactic complexity on users was not as great as initially hoped. There are two main reasons for this. In order to minimise the number of errors that could be introduced, the system integrated in OpenBook took a very conservative approach when applying rewriting rules. For this reason, a limited number of sentences were affected by processing. Secondly, when the rewriting rules generate correct sentences, users are not immediately aware that the text has been made more accessible. 
For this reason, we rarely found direct references to the benefits of reducing structural complexity in users' feedback. However, we believe this processing has contributed to the overall positive feedback received.

\subsection{Processing ambiguity in meaning}

A literature review together with research carried out in the project showed that the lexical component of language may be the one posing most difficulties for people with autism. Specific lexical items such as mental verbs, emotional language and figurative language constitute a difficult barrier to overcome for people with ASD. To address this issue, a set of LT modules were developed to detect semantic obstacles in input texts and, whenever possible, to resolve them. These modules, together with a collection of relevant resources were integrated into a framework for meaning disambiguation. While developing this framework, emphasis was, as much as possible, on the development of language independent modules. As can be seen in Table 3 the user requirements analysis revealed a large number of potential obstacles caused by semantic ambiguity. Given the difficulty of addressing these obstacles, the consortium agreed to focus on those with higher priority. For brevity, we present only these high priority obstacles here. They can be categorised into three broad groups: coreference, difficult words, and figurative language. The only aspect of emotional language that was tackled in the project is the one linked to mental verbs. The other recommendations, although marked as high priority, were not dealt with because they were considered too complicated to be reliably identified by an automatic system.

For all the obstacles, detection is first carried out. Then, depending on the type of obstacle, a different assistive element is provided, thus providing several strategies for the resolution of the obstacles. For example, for difficult words, the resolution comprises the extraction of definitions and synonyms of terms; for acronyms, their expanded form is provided; for infrequent slang, the expression is normalised, and for coreference, the antecedent of a pronoun, definite description, or omitted pronoun is provided.

In this section, we only provide a brief overview of the methods employed by the meaning disambiguator. A more detailed description is presented in the technical report by Lloret et al. [33].

3.4.1. Processing figurative language. Figurative language in general and idioms in particular present specific problems for our end users, as they are not able to grasp the meaning of these expressions. When reading a text they tend to construct the literal meaning of figurative expressions such as "calm before the storm" or "raining cats and dogs" and therefore misunderstand the meaning of the sentence that contains them. Even though some progress has been made in the field in recent years, the identification of conceptual metaphors for open domains is beyond the current state of the art in LT. For this reason the approach taken in the FIRST project to deal with figurative language was to compile dictionaries for each language. To allow more flexibility some of the entries in these dictionaries were encoded as JAPE 


\begin{tabular}{|c|c|c|}
\hline Obstacle & Text simplification operation & ID \\
\hline \multirow[t]{2}{*}{ Polysemy } & Provide easier synonyms. & UR401 \\
\hline & Detect and highlight polysemy. & UR425 \\
\hline \multirow{3}{*}{$\begin{array}{l}\text { Phraseological units } \\
\text { (idioms, lexicalised } \\
\text { metaphors) }\end{array}$} & Replace by a simple word. & UR402 \\
\hline & Detect and highlight when replacement is not possible. & UR425 \\
\hline & $\begin{array}{l}\text { Provide simple definitions to explain phraseological } \\
\text { units. }\end{array}$ & UR410 \\
\hline \multirow[t]{3}{*}{ Less common words } & Replace infrequent words with simpler synonym. & UR405 \\
\hline & Provide simple definitions to explain mental verbs. & UR411 \\
\hline & Provide simple definitions to explain infrequent words. & UR413 \\
\hline \multirow[t]{3}{*}{ Emotional language } & $\begin{array}{l}\text { Replace complicated emotional adjectives with simpler } \\
\text { synonyms. }\end{array}$ & UR404 \\
\hline & $\begin{array}{l}\text { Provide simple definitions to explain emotional } \\
\text { adjectives. }\end{array}$ & UR412 \\
\hline & Replace complex mental verbs with simpler synonyms. & UR403 \\
\hline \multirow[t]{4}{*}{ Slang } & Normalize infrequent slang. & UR407 \\
\hline & Provide simple definitions to explain infrequent slang. & UR414 \\
\hline & Detect specialized slang belonging to a domain. & UR423 \\
\hline & Provide simple definitions to explain specialized slang. & UR424 \\
\hline Infrequent acronyms & $\begin{array}{l}\text { Expand infrequent acronyms } \\
\text { and abbreviations }\end{array}$ & UR415 \\
\hline $\begin{array}{l}\text { Long numerical } \\
\text { expressions }\end{array}$ & Express long numerical expressions with digits. & UR417 \\
\hline Anaphors & $\begin{array}{l}\text { Detect and leave anaphors with low resolution } \\
\text { confidence unresolved. } \\
\text { Resolve pronominal anaphora. } \\
\text { Resolve definite descriptions. } \\
\text { Resolve ellipsis. }\end{array}$ & $\begin{array}{l}\text { UR418 } \\
\text { UR419 } \\
\text { UR420 } \\
\text { UR421 }\end{array}$ \\
\hline
\end{tabular}

TABLE 3. User requirements relating to the processing of ambiguity in meaning

expressions [15]. This allowed detection of accepted morphological variations of these expressions and cases involving discontinuous expressions. Lloret et al. [33] and Barbu et al. [2] present more details of the methods developed in the project to detect figurative expressions (idioms).

3.4.2. Processing difficult words. To process difficult words, such as polysemous words, acronyms, abbreviations and slang, the recommended strategy was to provide definitions and/or synonyms. However, depending on the nature of the obstacle, in some cases the definition and/or synonym is considered from a broader perspective. In the case of acronyms or infrequent slang, the framework provides an expansion of the acronym or the normalised version of the slang expression, respectively, as assistive elements to facilitate comprehension of them. A set of resources was compiled to support this. After analysing and evaluating the existing available LT resources, it was noticed that the accuracy of disambiguation of polysemous words, specialised slang, less common words, and mental verbs is limited by the use of WordNet and WordNet-related resources. 
3.4.3. Processing coreference. The analysis and research carried out into coreference resolution for Bulgarian, English, and Spanish resulted in the development of three modules capable of detecting and resolving: pronominal anaphora for Bulgarian; pronominal anaphora for English; and pronominal anaphora, definite descriptions and ellipsis for Spanish. The types of coreference phenomena that could be addressed in each language depended on the availability of tools and resources for that language. For Bulgarian, an extension of the MSTParser [62] was employed by the coreference resolution system. For English, the Stanford Deterministic Coreference Resolution System [32] was used since our evaluation showed that this was the best performing system and the most appropriate to be used in the context of the project. For Spanish, the process was divided into three stages, in order to separate the functionalities of anaphor detection; potential candidate antecedent identification; and, finally, anaphora resolution. In this manner, depending on the type of coreferential phenomenon to be resolved, a different approach was used. The coreference module integrated in Open Book relied on Freeling for the detection of pronominal anaphora and definite descriptions, the Naïve Bayes algorithm for detecting ellipsis, a Voted Feature Interval algorithm for resolving pronominal anaphora and ellipsis, and the PART algorithm for resolving definite descriptions.

The pronoun resolver for English was evaluated on texts of the news, literature and health registers. This evaluation showed that if used as a tool to aid in post-editing of texts, then for more than $90 \%$ of the anaphoric pronouns, the system is able to present a list of options which contain the antecedent. A small scale evaluation of the Bulgarian pronoun resolver showed that its accuracy is around $50 \%$. The evaluation results of the coreference resolver for Spanish both, for detection and resolution, shows that it achieves superior results to the best performing system in the Semeval-2010 task [47].

\subsection{Generation of personalised documents}

In addition to making the text more readable, an important aspect of making documents more accessible is the possibility to produce multimodal personalised documents. This is done by implementing modules which can supplement texts with images and summaries, and by providing a wide range of personalisable parameters derived from user requirements. The most important user requirements for this type of processing are presented in Table 4.

3.5.1. Generation of multimodal documents. Images are very useful for explaining the meaning of a word, regardless of whether they are polysemous or rare. For this reason, two image retrieval systems were implemented to retrieve images from expressions automatically identified as difficult: an offline image retrieval module and an online image retrieval module. The offline image retrieval method uses information from the disambiguation module used to deal with ambiguity in meaning (see Section 3.4) to map single words and multiword expression to images extracted from the ImageNet database [17]. This database stores web images annotated with WordNet noun synsets. The 


\begin{tabular}{|l|l|l|}
\hline Obstacle & Text simplification operation & ID \\
\hline $\begin{array}{l}\text { Understanding of } \\
\text { general meaning }\end{array}$ & $\begin{array}{l}\text { Give relevant idea on top of text. } \\
\text { Show key words. } \\
\text { Post questions in or after the text to help monitor } \\
\text { comprehension. } \\
\text { Give information on key concepts before reading text. } \\
\text { Support the overall meaning of the text with images } \\
\text { Provide text with summaries }\end{array}$ & $\begin{array}{l}\text { UR502 } \\
\text { UR503 } \\
\text { UR505 } \\
\text { UR506 }\end{array}$ \\
\hline Phraseological units & $\begin{array}{l}\text { Support the understanding of phraseological units } \\
\text { with images. }\end{array}$ & UR507 \\
\hline $\begin{array}{l}\text { Non lexicalized } \\
\text { metaphors }\end{array}$ & $\begin{array}{l}\text { Support the understanding of metaphorical } \\
\text { language with images. }\end{array}$ & UR508 \\
\hline Less common words & $\begin{array}{l}\text { Support the understanding less common words } \\
\text { with images. }\end{array}$ & UR509 \\
\hline Emotional language & $\begin{array}{l}\text { Support the understanding emotional adjectives } \\
\text { with images. }\end{array}$ & UR510 \\
\hline Polysemy & Support the understanding of polysemy with images. & UR511 \\
\hline
\end{tabular}

TABLE 4. User requirements relating to the generation of personalised documents

ImageNet database links around 22,000 synsets with more than 14 million images, each checked using crowdsourcing to ensure the corectness of the association. In addition, Wikification [37] is used to link terms in the text with their corresponding Wikipedia page, which in turn is used to extract images that explain the terms. In cases where the offline image retrieval engine is unable to identify an appropriate image, the online image retrieval module queries Google and Bing for images related to the term. In line with expectation, the accuracy of this type of image retrieval is much lower than that obtained by the offline module. Despite this, end users of the OpenBook system very much appreciated the implemented image retrieval functions.

3.5.2. Generation of summaries. Another way to help users of OpenBook is by preparing summaries of documents. OpenBook implements a sentence extraction algorithm inspired by TextRank [38] or LexRank [20] which identifies the most important sentences in the original text and builds a summary that includes only those important sentences, presented in the order in which they appeared in the original document. This algorithm was selected because it is language independent, it was deemed better than other general summarisation approaches, and it is fast. The size of the summary is controlled by a personalisable parameter and carers have the option to modify the summary to make it more comprehensive and to better fit users needs. Interviews with users (discussed in Section 4.3) revealed that, despite their relatively low accuracy, both image retrieval and automatic text summarisation were widely used and appreciated by carers and end users.

3.5.3. Personalisation. The personalisation of the produced documents can be controlled by users via a set of parameters decided on the basis of user requirements analysis. Users are able to control which LT components are run and to change their behaviour. For instance, users can indicate whether 
they want to obtain only definitions of words, rather than definitions and synonyms, or in the case of syntactic simplification indicate whether obstacles should only be detected or both detected and removed. End users and their carers can set these parameters for each individual document. Personalisation also includes parameters which control the look and feel of the interface, a very important aspect for users with ASD.

\section{Evaluation of the FIRST project}

The validation of the impact of the FIRST technology on inclusion was made through quantitative evaluation via reading comprehension testing and qualitative research methodologies employing semi-structured interview techniques to compare the perceptions of people with ASD, their family members, and other relevant intermediaries [25]. Specifically, FIRST technologies are designed for people with high-functioning autism, defined as those with a formal diagnosis of autism and an IQ score greater than 70. Intermediaries (carers) also participated in the evaluation. The usefulness of the OpenBook software was also assessed through a benchmarking experiment where the times taken for carers to simplify texts when they had access to the tool and when they had no access to it were recorded.

\subsection{Reading comprehension testing}

4.1.1. Setting of the experiment. Reading comprehension testing was used to test the effectiveness of OpenBook as a tool to convert texts into a more accessible form for end users. 243 participants (193 males and 50 females) with high-functioning autism were recruited in the UK, Spain and Bulgaria. In addition, a control group of 50 typical children were involved in the experiment in Bulgaria. Comprehension tests were conducted in a controlled environment under time-limited conditions. Each participant was presented with a battery of 6 texts followed by multiple-choice questions and a subjective text rating. Half of the texts were presented in their original form while the remainder were presented in a more accessible form generated by carers using OpenBook. The order of text presentation was random and both researchers and participants were blind to this order. The hypothesis investigated was that text simplification improves reading comprehension for participants with ASD, and therefore participants will be able to correctly answer more questions about converted texts than about texts in their original form.

Each clinical centre in the UK and Bulgaria identified 6 texts that were appropriate for respective age groups (adults and children) under examination. The research team in Spain identified 12 texts in total: 6 for children and 6 for adults. Texts for adults were selected from comprehension test batteries used to examine reading comprehension in proficient language learners. Texts for children were selected from childrens books and the internet. The texts for adults used in Spain and the UK were matched for word length and complexity. The same was done for the texts for children in Spain and Bulgaria. Text complexity was assessed using methods developed in the project and which 
focused on the obstacles identified in the user requirements. Additional evaluation of the readability of these texts, using both standard readability metrics and metrics predicted to be relevant for readers with ASD, was described in the paper by Yaneva and Evans [60].

The original texts were processed automatically using the OpenBook tool and then post-edited by the clinical teams, who acted as intermediaries for people with ASD. Post-editing operations were similar to those performed in the benchmarking experiment presented in the next section. Multiple-choice questions (MCQs) were generated by each clinical team for their respective texts, with the assistance of technical partners. MCQs were selected to test the general comprehension of the text, especially parts of the text containing identified obstacles to reading comprehension. Each adult text was followed by 6 MCQs, whilst children's texts had 4 MCQs. This selection was made to accommodate children's performance within the same timeframe as the adults. Both children and adults had 10 minutes per text to read and answer MCQs. Although we had planned to run reading comprehension sessions in large groups, we approached this activity with flexibility considering the social challenges and anxieties faced by people with ASD. Therefore, we ran the tests in smaller groups and sometimes in one-to-one sessions.

4.1.2. Evaluation results. The reading comprehension tests indicated that participants performed better with MCQs based on versions of texts converted using OpenBook than on the original versions of the same texts $(\mathrm{t}=4.42, \mathrm{p}<0.001$, CI $[0.63,0.79])$. However, the result was only of borderline statistical significance for Spanish children. Participants also rated blindly converted texts as easier to understand. $(t=6.96, \mathrm{p}<0.001$, CI $[0.71,1.26])$. This was consistent for UK, Spain and Bulgaria, for both adults and children.

Analysis of the data also suggests that there was no significant association between the comprehension scores and the subjective scores for either the scores for texts in their original form or the scores for texts in their simplified form. This means that although participants gave more correct answers in response to questions about texts converted using OpenBook, they did not identify them as being easier to understand. This suggests that the process of making text more accessible does not interfere with the way users regard the texts. A more detailed description of the testing procedure and discussion of the results is provided in the technical report of the project [28] and the paper by Jordanova and Cerga Pashoja [27]

\subsection{Evaluation of Text Conversion using OpenBook: Readability Assessment}

In order to assess the extent to which the OpenBook software reduces the burden on carers converting texts into a more accessible form for end users, a benchmarking experiment was carried out. This experiment was conducted in two stages. In the first year of the project, professional carers were asked to convert 25 heterogenous texts in Bulgarian, English, and Spanish into a more 


\title{
Instructions to professionals: How to Simplify Texts
}

\author{
You need to time yourself from the moment you start reading each text to the moment you \\ finish the simplification. This should be done separately for each text. \\ Should you need to interrupt the work, pause the timer and restart timing once you restart \\ simplification task. \\ $>$ Please do not read the texts beforehand. \\ $>$ Read the instructions below carefully. Do not start simplifications if you are not clear or sure \\ about any of the instructions (or parts of them). Please contact me if you do not understand \\ or need clarification about the instructions below before starting the task.

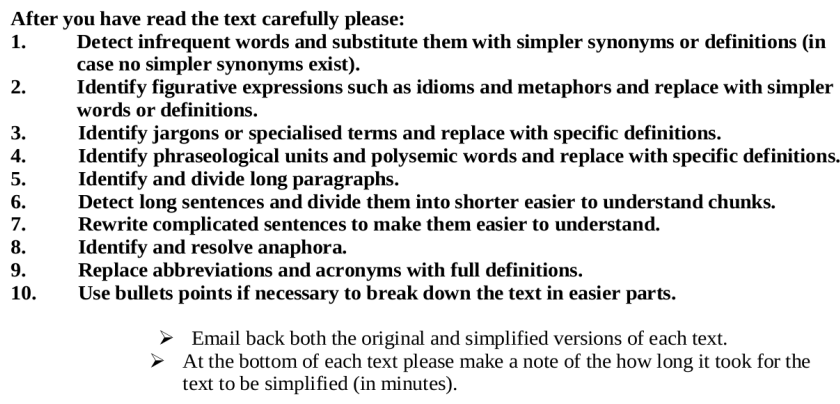

FiguRE 3. Text conversion guidelines

accessible form without the use of OpenBook. The second stage was semiautomatic in nature: carers used the interface to OpenBook when converting the texts, and were able to exploit LT functions to assist in the process. The same carers participated in both stages and the time it took them to perform the simplification conversion was recorded. Participants in the conversion task were provided with the guidelines shown in Fig. 3. The guidelines also provided definitions of the linguistic terms used.

The mean time taken to convert texts with 250-350 words decreased significantly from 54 minutes for unaided conversion to 29 minutes for conversion using OpenBook. The time increased only for English, but this increase is not statistically significant. When using OpenBook carers inserted images in the simplified document, a process that they did not attempt during unaided conversion as it was considered too onerous. During unaided conversion, huge disparities in conversion rates between centres and carers were noted. These disparities diminished when OpenBook was used.

4.2.1. Readability. In addition to analysing the time taken to convert the text, we also carried out an analysis of the readability of the texts in order to check that the converted ones are indeed more accessible. The readability of each version of the texts was assessed using eight language independent readability metrics sensitive to variables such as the frequency of occurrence of commas, pronouns, metaphors, passive verbs, and polysemic words in the texts, the lengths of words and sentences in the texts, and the type token ratio of the text. One language specific readability metric combining and weighting information about the language independent metrics was also used. In terms of readability, we made the following observations about texts produced via semi-automatic text conversion in which carers exploited OpenBook: 
- In Bulgarian, converted texts contain: fewer phraseological units and non-lexicalised metaphors than the originals (with statistical significance), and more polysemous words than the originals (with statistical significance), possibly due to increases in the diversity of vocabulary.

- In English, converted texts contain: fewer commas, shorter words, less diverse vocabulary, fewer phraseological units and non-lexicalised metaphors, and reduced syntactic complexity ${ }^{6}$

- In Spanish, converted texts contain: fewer metaphors, shorter sentences, less diverse vocabulary, more pronouns, and fewer polysemous words

We compared texts generated by carers exploiting OpenBook with texts generated by carers who were unaided. We noted that for Bulgarian texts, use of OpenBook led to smaller reductions in the numbers of phraseological units and non-lexicalised metaphors used, but smaller undersirable increases in the numbers of polysemous words used than was the case in unaided conversion. For English texts, use of OpenBook led to smaller reductions in the numbers of phraseological units and non-lexicalised metaphors and larger reductions in the diversity of vocabulary than unaided conversion. The explanation for use of OpenBook leading to smaller reductions in the occurrence of phraseological units and non-lexicalised metaphors in Bulgarian and English texts is that rather than re-phrasing and deleting these elements from sentences, as unaided editors do, OpenBook provides explanatory definitions of them. These figurative elements thus have a tendency to be preserved in the text. For Spanish texts, use of OpenBook led to larger reductions in sentence length and larger reductions in the number of metaphors used in the converted texts.

These findings are derived from information about readability metrics for which there were statistically significant differences between the scores for texts in their original and converted versions. More detailed analysis and explanation of the findings of these experiments are reported in the technical report by Jordanova et al. [28].

\subsection{User feedback}

Individual interviews were carried out in order to understand better the experiences of people using OpenBook and to explore its impact on better access to written information and improved social inclusion. Users with ASD and their carers were interviewed using questions related to topics deemed important for their social inclusion. The interviews lasted between 20 and 50 minutes and were recorded, transcribed and analysed using the Atlas.it data analysis package. ${ }^{7}$ The interviews were used to obtain feedback from users about a period spent accessing the user interface to OpenBook, in their own time at home to access texts of their own choosing. In this context, end users exploited the LT to make fully automatic conversion of texts.

\footnotetext{
${ }^{6}$ Measured using Scarborough's index of productive syntax [50].

${ }^{7}$ http://atlasti.com/
} 
Thematic analysis was the framework used to analyse interview transcripts. Thematic analysis is a principal technique used by qualitative researchers to analyse data. It is a method for identifying, analysing, and reporting patterns (themes) within the data. In the case of the analysis carried out in the FIRST project, these were related to the effect OpenBook had on people with ASD and on their social inclusion).

Our thematic analysis led to the derivation of eight themes emerging from user feedback about use of OpenBook. These themes concerned positive changes in independence with regard to:

1. Comprehension: Improved reading comprehension as a result of using OpenBook was widely reported. The positive impact in comprehending written texts was spontaneously described as "obvious" and "encouraging" by one user. Other users reported improved comprehension when accessing complex information such as reading about formulae and mathematical curves and improvements in understanding subtext.

2. Reading: Improved comprehension seemed to have an impact on participants reading skills. Improved reading abilities were reported by both adults with ASD and their carers. Adults with ASD reported that they were reading more as a result of using OpenBook and focusing better on reading. Individual carers reported improvements in the vocabulary of children with ASD.

3. Writing: One unintended impact of their use of Openbook was experienced by both adults and carers of children with ASD. They reported improvements in their writing skills, gaining confidence in writing emails and notes. Some participants began writing notes for the first time, while others felt more confident and consequently became more active writers.

4. Communication: Improved communication was another theme that emerged from our analysis, and was consistent for both adults and children. One carer of a child with ASD reported improvements in their sociability, while adults with ASD reported improvements in sociability, vocabulary, and increased use of complex phrases and sentences. One teacher observed a student with ASD overcoming his shyness and becoming more sociable.

5. Emotions: Improvements in comprehension and communication were factors which seemed to have a positive impact on the emotions of both children and adults with ASD. One mother stated that her sons behaviour had improved and that her son no longer became angry when he failed to understand what his mother was saying.

6. Self-efficacy and confidence: Carers talked appreciatively about the fact that OpenBook apeared to make users more self-sufficient and consequently self-confident in their ability to look independently for information and communicate with others. Children involved in the study were reported to study longer and more effectively. 
7. Relationships: In the interviews, children were reported to engage more frequently with their peers and carers and the quality of these interactions was consistently reported to have been enhanced. One teacher in Bulgaria stated that she expected her workload to be alleviated as a result of the student engaging independently with OpenBook. Adults also reported changes in relationships. To illustrate, one user explained that she started leaving notes for her work colleagues to facilitate her workload. Several users (adults and children) talked about having become less reliant on others since using OpenBook. However, some users felt that some professional carers (e.g. social workers) may be insufficiently computer literate to support their use of OpenBook.

8. Independence: The increased independence of users of OpenBook was a recurring theme throughout the interviews. Although some concern was expressed about the possibility of adults with ASD becoming more dependent on their carers, overall, statements described increased independence of both adults and children with ASD when accessing OpenBook and reading texts, when accessing technology, when reading physical books, and when writing notes and emails.

OpenBook was reported to have had a positive impact on reading comprehension of both adults and children with ASD. Improved reading comprehension seemed to improve the reading skills of people with ASD and their writing and communication abilities. Although some users found it disconcerting to use OpenBook, most users stated that it had a positive effect on their relationships, self-confidence and ultimately their independence. Full details of our thematic analysis and examples of user feedback addressing the eight themes are provided in the technical report by Jordanova et al. [28].

Generally, users said that the system is easy to use and not only improves the studies of children with ASD and relieves anxiety of adults with ASD regarding text comprehension but it also relieves the burden on teachers and carers. Users said that they would like to continue to use OpenBook in the future and will recommend it to friends and colleagues.

However, OpenBook did not work well for all users. One adult with autism found it difficult to process the obstacles highlighted by the software. His mother explained that he was made uncomfortable by the font colour used to highlight obstacles to reading comprehension and felt he had done something wrong. Some users criticised OpenBook due to the relatively low accuracy of some components. They commented on the tendency of the image retrieval tool to return inappropriate images for some ambiguous words and substitution of some ambiguous words with more complex definitions and synonyms. Most of these issues were resolved through the development phase of the project, and users reported improvement in processing times and synonym suggestions. 


\section{Discussion and conclusions}

This paper has presented a project which brought together a consortium of nine partners from academic, industrial, health, and charity sectors to develop ICT to convert electronic documents into a form facilitating reading comprehension for people with ASD. Research was conducted to gain insight into the specific user requirements of end users with ASD and the intermediaries working with end users to help them in accessing information in texts. The findings of this study were used to underpin the development of a tool integrating language technology components to convert texts into a more accessible form. LT was developed in the project to reduce the structural complexity caused by long and syntactically complex sentences and the ambiguity in meaning caused by difficult/rare terms, ambiguous words, anaphora, and figurative language. An LT component was also developed to generate additional content such as concise summaries and images to explain complex terms occurring in texts that end users seek to access.

Evaluation of OpenBook was complex and user-focused, exploiting qualitative and quantitative methods to assess the tool intrinsically and extrinsically. Intrinsic evaluation of the LT services integrated in OpenBook revealed that they were not accurate enough to support fully automatic conversion of text into a form facilitating reading comprehension for people with ASD. For this reason, those services are delivered by two different interfaces, each supporting a different text conversion service. In the first, end users (people with ASD) apply a restricted set of reliable LT components to automatically improve the accessibility of texts they are seeking to read. In the second, intermediaries (carers, educators, and health service providers) have access to the full set of LT components which can assist them in converting texts into a more accessible form for end users.

The first conversion service was evaluated by analysis of feedback from end users. The interface was found to be easy to use and there was enthusiasm for the concept underlying it. LT components providing users with explanations of complex words and idioms, retrieving images to explain those concepts, and generating summaries of input texts were all valued by end users (and carers). There was some criticism of the inaccuracy of some functions and the poor handling/lack of coverage of some domain specific terms.

The second service was evaluated by assessing the extent to which the use of OpenBook to convert texts improves the comprehension of converted texts by end users and by examining differences between the process of converting texts into a more accessible form using OpenBook and the process of making the conversion in an unaided fashion. Reading comprehension testing showed that texts converted by carers using OpenBook were understood better than texts in their original form. As an editing tool, it was found that OpenBook enabled more rapid conversion of text to a more accessible form. The texts converted using OpenBook were not significantly less readable than those generated more slowly as a result of onerous unaided conversion and were significantly more readable than the originals. 
Overall, users recommended the use of OpenBook and were enthusiastic about using the system independently. They perceived that it made them more independent and keen to solve problems for themselves. Interviews with carers and end users revealed improvements in users' ability to comprehend texts, including improvements in understanding of subtext. Overall, use of OpenBook was associated with greater motivation to read, greater engagement in reading, and improved attention in reading as well as improvements in vocabulary. Improvements were also noted in the writing skills of both young and mature end users. Positive changes were also observed with regard to behaviour, communication and sociability of end users. There were anecdotal reports of collateral improvements in educational achievement.

One exciting aspect of the FIRST project is that the simplifying language technology developed can have wide-ranging applications beyond that of improving text accessibility for users with ASD. The software has potential to benefit other types of health service user, as well as groups in other sectors (e.g. language learners, migrants and readers of legal documentation). Certain LT components, such as the syntactic simplification module, were perceived to be of limited benefit for users in the FIRST project. However, the functionality of this module was restricted in accordance with the requirements of people with ASD and their low tolerance for errors in the system output. In its unrestricted mode, it is able to transform a wider range of syntactic constructions than was attempted in the project. We believe that these other types of transformation operation are applicable to other language processing applications, such as information extraction and text summarisation.

\section{Acknowledgment}

We would like to acknowledge the contribution of all the partners to the project. This paper would not have been possible without their contribution to the various stages of the research carried out. We would also like to thank the carers and individuals with high-functioning autism who participated in the different evaluations reported in this paper. The research was partially funded by the EC under the 7th Framework Programme for Research and Technological Development (FP7- ICT-2011.5.5 FIRST 287607)

\section{References}

[1] Angrosh, M., Nomoto, T., Siddharthan, A.: Lexico-syntactic text simplification and compression with typed dependencies. In: Proceedings of COLING 2014, the 25th International Conference on Computational Linguistics: Technical Papers, pp. 1996-2006 (2014)

[2] Barbu, E., Martín-Valdivia, M., Alfonso, L., Lopez, U.: Open book: a tool for helping asd users' semantic comprehension. In: Proceedings of the 2th Workshop of Natural Language Processing for Improving Textual Accessibility (NLP4ITA), pp. 11-19. Atlanta, US (2013)

[3] Barbu, E., Martín-Valdivia, M.T., Martínez-Cámara, E., na López, L.A.U.: Language technologies applied to document simplification for helping autistic 
people. Expert Systems with Applications: An International Journal 42(12), 5076-5086 (2015)

[4] Barzilay, R., Elhadad, N.: Sentence alignment for monolingual comparable corpora. In: Proceedings of the 2003 Conference on Empirical Methods in Natural Language Processing (EMNLP '03), pp. 25-32. Sapporo, Japan (2003)

[5] Bautista, S., Saggion, H.: Can numerical expressions be simpler? implementation and demostration of a numerical simplification system for spanish. In: Proceedings of the Ninth International Conference on Language Resources and Evaluation (LREC-2014) (2014)

[6] Biran, O., Brody, S., Elhadad, N.: Putting it simply: a context-aware approach to lexical simplification. In: Proceedings of the 49th Annual Meeting of the Association for Computational Linguistics: shortpapers (ACL-2011), pp. 496501. Portland, Oregon (2011)

[7] Bosma, W.: Image retrieval supports multimedia authoring, pp. 89-94. ITCirst, Trento, Italy (2005)

[8] Bott, S., Rello, L., Drndarevic, B., Saggion, H.: Can spanish be simpler? LexSiS: Lexical simplification for spanish. In: Proceedings of the 12th International Conference on Intelligent Text Processing and Computational Linguistics, Lecture Notes in Computer Science, pp. 8-15. Springer, Samos, Greece (2012)

[9] Brugha, T., McManus, S., Meltzer, H., Smith, J., Scoth, F.J., Purdon, S.: Autism spectrum disorders in adults living in households throughout england. Report from the Adult Psychiatric Morbidity Survey (2007)

[10] Canning, Y.: Syntactic simplification of text. Ph.d. thesis, University of Sunderland (2002)

[11] Chandrasekar, R., Srinivas, B.: Automatic induction of rules for text simplification. Knowledge-Based Systems 10, 183-190 (1997)

[12] Chen, H.B., Huang, H.H., Chen, H.H., Tan, C.T.: A simplification-translationrestoration framework for cross-domain smt applications. In: Proceedings of COLING 2012, pp. 545-560 (2012)

[13] Cohn, T., Lapata, M.: Sentence Compression as Tree Transduction. Journal of Artificial Intelligence Research 20(34), 637-74 (2009)

[14] Coster, W., Kauchak, D.: Simple english wikipedia: A new text simplification task. In: Proceedings of the 49th Annual Meeting of the Association for Computational Linguistics (ACL-2011), pp. 665-669. Portland, Oregon (2011)

[15] Cunningham, H., Maynard, D., Bontcheva, K., Tablan, V., Aswani, N., Roberts, I., Gorrell, G., Funk, A., Roberts, A., Damljanovic, D., Heitz, T., Greenwood, M.A., Saggion, H., Petrak, J., Li, Y., Peters, W.: Text Processing with GATE (Version 6) (2011). URL http://tinyurl.com/gatebook

[16] De Belder, J., Deschacht, K., Moens, M.F.: Lexical simplfication. In: Proceedings of the 1st International Conference on Interdisciplinary Research on Technology, Education, and Communication (Itrec-2010). Kortrijk, Belgium (2010)

[17] Deng, J., Dong, W., Socher, R., Li, L.J., Li, K., Fei-Fei, L.: ImageNet: A LargeScale Hierarchical Image Database. In: CVPR09 (2009)

[18] Dornescu, I., Evans, R., Orasan, C.: A Tagging Approach to Identify Complex Constituents for Text Simplification. In: Proceedings of Recent Advances in Natural Language Processing, pp. 221 - 229. Hissar, Bulgaria (2013) 
[19] Elhadad, N.: Comprehending technical texts: predicting and defining unfamiliar terms. In: AMIA Annual Symposium Proceedings, pp. 239-243 (2006)

[20] Erkan, G., Radev, D.R.: Lexrank: Graph-based lexical centrality as salience in text summarization. Journal of Artificial Intelligence Research 22(1), 457-479 (2004)

[21] Evans, R.: Comparing methods for the syntactic simplification of sentences in information extraction. Literary and Linguistic Computing 26 (4), 371-388 (2011)

[22] Evans, R., Orasan, C., Dornescu, I.: An evaluation of syntactic simplification rules for people with autism. In: Proceedings of the 3rd Workshop on Predicting and Improving Text Readability for Target Reader Populations (PITR), pp. 131-140. Gothenburg, Sweden (2014)

[23] Frith, U., Snowling, M.: Reading for meaning and reading for sound in autistic and dyslexic children. Journal of Developmental Psychology 1, 329-342 (1983)

[24] González-Navarro, A., Freire-Prudencio, S., Gil, D., Martos-Pérez, J., Jordanova, V., Cerga-Pashoja, A., Shishkova, A., Evans, R.: FIRST: una herramienta para facilitar la comprensión lectora en el trastorno del espectro autista de alto funcionamiento. Revista de Neurologia 58 (Supl 1), 129-135 (2014)

[25] Granizo, L., Naylor, P., del Barrio, C.: Analysis of the social relationships of pupils with asperger syndrome in mainstream secondary schools: case studies. Revista de Psicodidactica pp. 281-292 (2006)

[26] Jelínek, T.: Improvements to dependency parsing using automatic simplification of data. In: Proceedings of the Ninth International Conference on Language Resources and Evaluation (LREC-2014) (2014)

[27] Jordanova, V., Cerga Pashoja, A.: Effects of syntactic complexity, semantic reversibility, and explicitness on discourse comprehension in persons with aphasia and in healthy controls. BAOJ Psychology 1(003) (2016)

[28] Jordanova, V., Evans, R., Cerga Pashoja, A.: D7.8: Final evaluation report. Technical Report FIRST_D7.8_20141119, The FIRST Consortium (2014)

[29] Kajiwara, T., Yamamoto, K.: Evaluation dataset and system for japanese lexical simplification. In: Proceedings of the ACL-IJCNLP 2015 Student Research Workshop, pp. 35-40 (2015)

[30] Kandula, S., Curtis, D., Zeng-Treitler, Q.: A semantic and syntactic text simplification tool for health content. In: AMIA Annual Symposium Proceedings, pp. 366-370 (2010)

[31] Lafferty, J., McCallum, A., Pereira, F.C.: Conditional random fields: Probabilistic models for segmenting and labeling sequence data. In: Proc. 18th International Conf. on Machine Learning, pp. 282-289. Morgan Kaufmann (2001)

[32] Lee, H., Peirsman, Y., Chang, A., Chambers, N., Surdeanu, M., Jurafsky, D.: Stanford's multi-pass sieve coreference resolution system at the conll-2011 shared task. In: Proceedings of the Fifteenth Conference on Computational Natural Language Learning: Shared Task, CONLL Shared Task '11, pp. 2834. Association for Computational Linguistics, Stroudsburg, PA, USA (2011)

[33] LLoret, E., Moreda, P., Moreno, I., Canales, L.: D4.1: Meaning Disambiguator: v2.1. Technical Report FIRST_D4.1_20140531, The FIRST Consortium (2014) 
[34] Martos, J., Freire, S., González, A., Gil, D., Evans, R., Jordanova, V., Cerga, A., Shishkova, A., Orasan, C.: User preferences: Updated. Tech. Rep. D2.2, Deletrea, Madrid, Spain (2013)

[35] Max, A.: Syntactic simplification - an application to text for aphasic readers. Mphil in computer speech and language processing, University of Cambridge, Wolfson College (2000)

[36] McDonald, R.T., Nivre, J.: Analyzing and integrating dependency parsers. Computational Linguistics 37(1), 197-230 (2011)

[37] Mihalcea, R., Csomai, A.: Wikify!: Linking documents to encyclopedic knowledge. CIKM 7, 233-242 (2007)

[38] Mihalcea, R., Tarau, P.: Textrank: Bringing order into texts. In: D. Lin, D. Wu (eds.) Proceedings of EMNLP 2004, pp. 404-411. Association for Computational Linguistics, Barcelona, Spain (2004)

[39] Minshew, N., Goldstein, G.: Autism as a disorder of complex information processing. Mental Retardation and Developmental Disability Research Review 4, 129-136 (1998)

[40] Mishra, K., Soni, A., Sharma, R., Sharma, D.: Exploring the effects of sentence simplification on hindi to english machine translation system. In: Proceedings of the Workshop on Automatic Text Simplification - Methods and Applications in the Multilingual Society (ATS-MA 2014), pp. 21-29 (2014)

[41] Nation, K., Clarke, P., Wright, B., Williams, C.: Patterns of reading ability in children with autism-spectrum disorder. Journal of Autism \& Developmental Disorders 36, 911-919 (2006)

[42] O'Connor, I.M., Klein, P.D.: Exploration of strategies for facilitating the reading comprehension of high-functioning students with autism spectrum disorders. Journal of Autism and Developmental Disorders 34:2, 115-127 (2004)

[43] OECD: Literacy in the information age: Final report of the international adult literacy survey. Tech. rep., Organisation for Economic Co-operation and Development, Paris, France (2000)

[44] Ogden, C.K.: Basic English: a general introduction with rules and grammar. K. Paul, Trench, Trubner \& Co., Ltd., London (1932)

[45] Paetzold, G.: Reliable lexical simplification for non-native speakers. In: Proceedings of the 2015 Conference of the North American Chapter of the Association for Computational Linguistics: Student Research Workshop, pp. 9-16 (2015)

[46] Pellow, D., Eskenazi, M.: An open corpus of everyday documents for simplification tasks. In: Proceedings of the 3rd Workshop on Predicting and Improving Text Readability for Target Reader Populations (PITR), pp. 84-93. Gothenburg, Sweden (2014)

[47] Recasens, M., Màrquez, L., Sapena, E., Martí, M.A., Taulé, M., Hoste, V., Poesio, M., Versley, Y.: Semeval-2010 task 1: Coreference resolution in multiple languages. In: Proceedings of the 5th International Workshop on Semantic Evaluation, SemEval@ACL 2010, pp. 1-8. Uppsala, Sweden (2010)

[48] Rello, L., Baeza-Yates, R., Bott, S., Saggion, H.: Simplify or help?: Text simplification strategies for people with dyslexia. In: Proceedings of the 10th International Cross-Disciplinary Conference on Web Accessibility, pp. 15:1-15:10. New York, NY, USA (2013) 
[49] Rindflesch, T.C., Rajan, J.V., Hunter, L.: Extracting molecular binding relationships from biomedical text. In: Proceedings of the sixth conference on Applied natural language processing, pp. 188-195. Seattle, Washington (2000)

[50] Scarborough, H.S.: Index of productive syntax. Applied Psycholinguistics 11, $1-22(1990)$

[51] Siddharthan, A.: Syntactic simplification and text cohesion. Research on Language and Computation 4:1, 77-109 (2006)

[52] Siddharthan, A.: Text simplification using typed dependencies: A comparision of the robustness of different generation strategies. In: Proceedings of the 13th European Workshop on Natural Language Generation, pp. 2-11 (2011)

[53] Specia, L.: Translating from complex to simplified sentences. In: Proceedings of the Conference on Computational Processing of the Portuguese Language, pp. 30-39. Springer, Porto Alegre, RS, Brazil (2010)

[54] Sutton, C., McCallum, A.: An introduction to conditional random fields. arXiv preprint arXiv:1011.4088 (2010)

[55] Vickrey, D., Koller, D.: Sentence simplification for semantic role labeling. In: Proceedings of ACL-08: HLT, pp. 344-352 (2008)

[56] Štajner, S.: New data-driven approaches to text simplification. Ph.d. thesis, University of Wolverhampton (2015)

[57] Walker, A., Siddharthan, A., Starkey, A.: Investigation into human preference between common and unambiguous lexical substitutions. In: Proceedings of the 13th European Workshop on Natural Language Generation (ENLG), pp. 176-180. Nancy, France (2011)

[58] Wubben, S., van den Bosch, A., Krahmer, E.: Sentence simplification by monolingual machine translation. In: Proceedings of the 50th Annual Meeting of the Association for Computational Linguistics (ACL-12), pp. 1015-1024. Jeju, Republic of South Korea (2012)

[59] Xu, W., Callison-Burch, C., Napoles, C.: Problems in current text simplification research: New data can help. Transactions of the Association for Computational Linguistics 3, 283-297 (2015)

[60] Yaneva, V., Evans, R.: Six good predictors of autistic text comprehension. In: Proceedings of Recent Advances in Natural Language Processing (RANLP 2015), pp. 697-706. Hissar, Bulgaria (2015)

[61] Zeng-Treitler, Q., Goryachev, S., Kim, H., Keselman, A., Rosendale, D.: Making texts in electronic health records comprehensible to consumers: A prototype translator. In: AMIA Annual Symposium Proceedings, pp. 846-850 (2007)

[62] Zhikov, V., Georgiev, G., Simov, K., Osenova, P.: Combining POS tagging, dependency parsing and co-referential resolution for bulgarian. In: Proceedings of RANLP'13. Bulgaria (2013)

[63] Zhu, Z., Bernhard, D., Gurevych, I.: A monolingual tree-based translation model for sentence simplification. In: Proceedings of the 23rd International Conference on Computational Linguistics (Coling 2010), pp. 1353-1361. Beijing, China (2010) 


\section{Constantin Orăsan}

Research Institute in Information and Language Processing University of Wolverhampton, Wolverhampton, WV1 1LY, United Kingdom e-mail: C.Orasan@wlv.ac.uk

Richard Evans

Research Institute in Information and Language Processing University of Wolverhampton, Wolverhampton, WV1 1LY, United Kingdom e-mail: R.J.Evans@wlv.ac.uk

Ruslan Mitkov

Research Institute in Information and Language Processing University of Wolverhampton, Wolverhampton, WV1 1LY, United Kingdom e-mail: R.Mitkov@wlv.ac.uk 